\title{
The solutions of time and space conformable fractional heat equations with conformable Fourier transform
}

\author{
Yücel Çenesiz \\ Department of Mathematics, \\ Faculty of Science, \\ Selçuk University, Turkey \\ email: ycenesiz@selcuk.edu.tr, \\ ycenesiz@ymail.com
}

\author{
Ali Kurt \\ Department of Mathematics, \\ Faculty of Science and Art, \\ Mustafa Kemal University, Turkey \\ email: alikurt@mku.edu.tr
}

\begin{abstract}
In this paper our aim is to find the solutions of time and space fractional heat differential equations by using new definition of fractional derivative called conformable fractional derivative. Also based on conformable fractional derivative definition conformable Fourier Transform is defined. Fourier sine and Fourier cosine transform definitions are given and space fractional heat equation is solved by conformable Fourier transform.
\end{abstract}

\section{Introduction}

Fractional differential equations which are the generalization of differential equations are successful models of real life events and have many applications in various fields in science [1]-[8]. So the subject becomes very captivating. Hence, many researchers have been trying to form a new definition of fractional derivative. Most of these definitions include integral form for fractional derivatives. Two of these definitions which are most popular:

2010 Mathematics Subject Classification: 26A33, 35R11, 34A08

Key words and phrases: time fractional heat equation, space fractional heat equation, conformable fractional derivative, conformable Fourier transform 
1. Riemann-Liouville definition: If $n$ is a positive integer and $\alpha \in[n-1, n)$, $\alpha$ derivative of $f$ is given by

$$
D_{a}^{\alpha}(f)(t)=\frac{1}{\Gamma(n-\alpha)} \frac{d^{n}}{d t^{n}} \int_{a}^{t} \frac{f(x)}{(t-x)^{\alpha-n+1}} d x .
$$

2. Caputo definition: If $n$ is a positive integer and $\alpha \in[n-1, n), \alpha$ derivative of $f$ is given by

$$
D_{a}^{\alpha}(f)(t)=\frac{1}{\Gamma(n-\alpha)} \int_{a}^{t} \frac{f^{(n)}(x)}{(t-x)^{\alpha-n+1}} d x .
$$

In $[9,10] \mathrm{R}$. Khalil and et al. give a new definition of fractional derivative called "conformable fractional derivative".

Definition 1 Let $\mathrm{f}:[0, \infty) \rightarrow \mathrm{R}$ be a function. $\alpha^{\text {th }}$ order conformable fractional derivative of $\mathrm{f}$ is defined by

$$
T_{\alpha}(f)(t)=\lim _{\varepsilon \rightarrow 0} \frac{f\left(t+\varepsilon t^{1-\alpha}\right)-f(t)}{\varepsilon}
$$

for all $\mathrm{t}>0, \alpha \in(0,1)$. If $\mathrm{f}$ is $\alpha$-differentiable in some $(0, \mathrm{a}), \mathrm{a}>0$, and $\lim _{\mathrm{t} \rightarrow 0^{+}} \mathrm{f}^{(\alpha)}(\mathrm{t})$ exists, then define

$$
f^{(\alpha)}(0)=\lim _{t \rightarrow 0^{+}} f^{(\alpha)}(t) .
$$

This new definition satisfies the properties which are given in the following theorem $[9,10]$.

Theorem 1 Let $\alpha \in(0,1]$ and $\mathrm{f}, \mathrm{g}$ be $\alpha$-differentiable at point $\mathrm{t}>0$. Then

(a) $T_{\alpha}(c f+d g)=c T_{\alpha}(f)+d T_{\alpha}(g)$, for all $a, b \in R$.

(b) $\mathrm{T}_{\alpha}\left(\mathrm{t}^{\mathrm{p}}\right)=\mathrm{pt}^{\mathrm{p}-\alpha}$ for all $\mathrm{p} \in \mathrm{R}$.

(c) $\mathrm{T}_{\alpha}(\lambda)=0$ for all constant functions $\mathrm{f}(\mathrm{t})=\lambda$.

(d) $T_{\alpha}(f g)=f T_{\alpha}(g)+g T_{\alpha}(f)$.

(e) $T_{\alpha}\left(\frac{f}{g}\right)=\frac{g T_{\alpha}(g)-f T_{\alpha}(f)}{g^{2}}$. 
(f) If, in addition to $f$ is differentiable, then $\mathrm{T}_{\alpha}(\mathrm{f})(\mathrm{t})=\mathrm{t}^{1-\alpha} \frac{\mathrm{df}}{\mathrm{dt}}$.

In Section 2, we will give the solution of fractional heat equation for $0<$ $\alpha<1$ with the help of conformable fractional derivative definition. In Section 3 , we will give conformable Fourier transform, conformable Fourier sine and cosine transform definitions and solve the space fractional heat equation with this transform.

\section{Time fractional heat equation}

General form for one dimension heat equation is

$$
\frac{\partial u}{\partial t}=\kappa \frac{\partial^{2} u}{\partial x^{2}} .
$$

Heat equation has many fractional forms. In this paper we investigate the solution of time fractional heat differential equation:

$$
\frac{\partial^{\alpha} u}{\partial t^{\alpha}}=\kappa \frac{\partial^{2} u}{\partial x^{2}}, 0<x<L, t>0
$$

with conditions

$$
\begin{gathered}
u(0, t)=0, t \geq 0 \\
u(L, t)=0, t \geq 0 \\
u(x, 0)=f(x), 0 \leq x \leq L
\end{gathered}
$$

where the derivative is conformable fractional derivative and $0<\alpha<1$.

Firstly we can mention conformable fractional linear differential equations with constant coefficients

$$
\frac{\partial^{\alpha} y}{\partial t^{\alpha}} \pm \mu^{2} y=0
$$

From formula (f) in Theorem 1 we can obtain

$$
\frac{\partial^{\alpha} y}{\partial t^{\alpha}}=t^{1-\alpha} \frac{d y}{d t}
$$

By substituting (6) in (5) it becomes following first order linear differential equation

$$
t^{1-\alpha} \frac{d y}{d t} \pm \mu^{2} y=0
$$


One can easily see that the solution of equation (7)

$$
y=c e^{\frac{ \pm \mu^{2}}{\alpha} t^{\alpha}} .
$$

Now we can use separation of variables method [11] for solution of our time fractional heat equation (1). Let $u=P(x) Q(t)$. Substituting this equation in Eq. (1), we have

$$
\frac{d^{\alpha} Q(t)}{d t^{\alpha}} P(x)=\kappa \frac{d^{2} P(x)}{d x^{2}} Q(t)
$$

from which we obtain

$$
\frac{d^{\alpha} Q(t)}{d t^{\alpha}} / \kappa Q(t)=\frac{d^{2} P(x)}{d x^{2}} / P(x)=\omega .
$$

As a result:

$$
\frac{d^{\alpha} Q(t)}{d t^{\alpha}}-\omega \kappa Q(t)=0
$$

and

$$
\frac{d^{2} P(x)}{d x^{2}}-\omega P(x)=0 .
$$

Now, we think about the equation

$$
\frac{d^{2} P(x)}{d x^{2}}-\omega P(x)=0 .
$$

For this equation, there are three cases for values of $\omega$ to be evaluated. $\omega=$ $0, \omega=-\mu^{2}, \omega=\mu^{2}$.

Conditions (2) and (3) give

$$
\mu=\frac{n \pi}{L} \text { and } P_{n}(x)=a_{n} \sin \frac{n \pi x}{L} .
$$

Equations (5) and (8) give,

$$
\mathrm{Q}_{\mathrm{n}}(\mathrm{t})=\mathrm{b}_{\mathrm{n}} e^{-\left(\frac{\mathrm{n} \pi}{\mathrm{L}}\right)^{2} \frac{\mathrm{K}}{\alpha} \mathrm{t}^{\alpha}} .
$$

Then, using the equations (9) and (10) the solution of the Cauchy problem which satisfies two boundary conditions obtained as

$$
u(x, t)=\sum_{n=1}^{\infty} c_{n} \sin \frac{n \pi x}{L} e^{-\left(\frac{n \pi}{L}\right)^{2} \frac{k}{\alpha} t^{\alpha}} .
$$


With the help of condition (4)

$$
c_{n}=\frac{2}{L} \int_{0}^{L} f(x) \sin \left(\frac{n \pi x}{L}\right) d x .
$$

Substituting (12) in (11) we find the solution as

$$
u(x, t)=\sum_{n=1}^{\infty} \sin \frac{n \pi x}{L} e^{-\left(\frac{n \pi}{L}\right)^{2} \frac{k}{\alpha} t^{\alpha}}\left[\frac{2}{L} \int_{0}^{L} f(x) \sin \left(\frac{n \pi x}{L}\right) d x\right] .
$$

\section{Conformable Fourier transform}

In [12] Abdeljawad gave the definition of conformable Laplace transform and in [13] Negero made a study on application of Fourier transform to partial differential equations. Now in this section we define conformable Fourier transform, infinite and finite Fourier sine and cosine transform. We give some properties of this transforms. At the end we use finite Fourier sine transform to solve space fractional heat equation.

Definition 2 Let $0<\alpha \leq 1$ and $\mathrm{h}(\mathrm{x})$ is real valued function defined on $(-\infty, \infty)$. The conformable Fourier transform of $\mathrm{h}(\mathrm{x})$ which is denoted by $\mathrm{F}_{\alpha}\{\mathrm{h}(\mathrm{t})\}(\boldsymbol{w})$ is given by

$$
F_{\alpha}\{h(t)\}(w)=H_{\alpha}(w)=\frac{1}{\sqrt{2 \pi}} \int_{-\infty}^{\infty} e^{-i w \frac{t^{\alpha}}{\alpha}} h(t) t^{\alpha-1} d t .
$$

Theorem 2 Let $0<\alpha \leq 1$ and $\mathrm{h}(\mathrm{x})$ is $\alpha$-differentiable real valued function defined on $(-\infty, \infty)$. Then

$$
\mathrm{F}_{\alpha}\left\{\mathrm{T}_{\alpha}(\mathrm{h})(\mathrm{t})\right\}(w)=i w \mathrm{H}_{\alpha}(w) .
$$

Proof. The proof followed by Theorem 1 (f) and known integration by parts.

Lemma 1 Let $\mathrm{f}:(-\infty, \infty) \rightarrow \mathrm{R}$ be a function which satisfies $\mathrm{F}_{\alpha}\{\mathrm{h}(\mathrm{t}), \boldsymbol{w}\}=$ $\mathrm{H}_{\alpha}(w)$ property. Then,

$$
F_{\alpha}\{h(t)\}(w)=F\left\{h\left((\alpha t)^{\frac{1}{\alpha}}\right)\right\}(w)
$$


where $F\{h(t)\}(w)=\frac{1}{\sqrt{2 \pi}} \int_{-\infty}^{\infty} e^{-i w t} h(t) d t$.

Proof. One can prove it easily by setting $t=\frac{\mathfrak{u}^{\alpha}}{\alpha}$.

Lemma $2 \mathrm{~F}_{\alpha}\{\mathrm{h}(\mathrm{t})\}(\boldsymbol{w})$ Fourier transform is a linear operator.

$$
F_{\alpha}\{a f+b g\}=a F_{\alpha}\{f\}+b F_{\alpha}\{g\} .
$$

Theorem 3 (Convolution Theorem). Let $\mathrm{g}(\mathrm{t})$ and $\mathrm{h}(\mathrm{t})$ be arbitrary functions. Then

$$
\mathrm{F}_{\alpha}\{\mathrm{g} * \mathrm{~h}\}=\sqrt{2 \pi} \mathrm{F}_{\alpha}\{\mathrm{g}\} \mathrm{F}_{\alpha}\{\mathrm{h}\}
$$

where $\mathrm{g} * \mathrm{~h}$ is the convolution of functions $\mathrm{g}(\mathrm{t})$ and $\mathrm{h}(\mathrm{t})$ defined as

$$
(g * h)(t)=\int_{-\infty}^{\infty} g(x) h(t-x) d x=\int_{-\infty}^{\infty} g(t-x) h(x) d x
$$

Proof. From Lemma 1, by using definition and changing the order of integration, we get

$$
\begin{aligned}
F_{\alpha}\{(g * h)(t)\} & =F\left\{(g * h)\left((\alpha t)^{\frac{1}{\alpha}}\right)\right\} \\
& =\frac{1}{\sqrt{2 \pi}} \int_{-\infty}^{\infty} \int_{-\infty}^{\infty} g\left((\alpha x)^{\frac{1}{\alpha}}\right) h\left((\alpha(t-x))^{\frac{1}{\alpha}}\right) e^{-i w t} d x d t \\
& =\frac{1}{\sqrt{2 \pi}} \int_{-\infty}^{\infty} \int_{-\infty}^{\infty} g\left((\alpha x)^{\frac{1}{\alpha}}\right) h\left((\alpha(t-x))^{\frac{1}{\alpha}}\right) e^{-i w t} d t d x .
\end{aligned}
$$

By making substitution $t-x=v$, so $t=v+x$,

$$
\begin{aligned}
F_{\alpha}\{(g * h)(t)\} & =\frac{1}{\sqrt{2 \pi}} \int_{-\infty}^{\infty} \int_{-\infty}^{\infty} g\left((\alpha x)^{\frac{1}{\alpha}}\right) h\left((\alpha v)^{\frac{1}{\alpha}}\right) e^{-i w(v+x)} d v d x \\
& =\frac{1}{\sqrt{2 \pi}} \int_{-\infty}^{\infty} g\left((\alpha x)^{\frac{1}{\alpha}}\right) e^{-i w x} d x \int_{-\infty}^{\infty} h\left((\alpha v)^{\frac{1}{\alpha}}\right) e^{-i w v} d v \\
& =\sqrt{2 \pi} F_{\alpha}\{g\} F_{\alpha}\{h\} .
\end{aligned}
$$




\section{Conformable Fourier transform of partial derivatives}

Lemma 3 For given $\mathrm{u}(\mathrm{x}, \mathrm{t})$ with $-\infty<\mathrm{x}<\infty$ and $\mathrm{t}>0$, we have

i. $F_{\alpha}\left\{\frac{\delta}{\delta t}(u(x, t))\right\}(w)=\frac{d}{d t} \widehat{u}(w, t)$.

ii. $F_{\alpha}\left\{\frac{\delta^{n}}{\delta t^{n}}(u(x, t))\right\}(w)=\frac{d^{n}}{d t^{n}} \widehat{u}(w, t), n=1,2,3, \ldots$

iii. $\mathrm{F}_{\alpha}\left\{\mathrm{T}_{\alpha}(\mathrm{u}(\mathrm{x}, \mathrm{t}))\right\}(w)=\mathfrak{i} w \widehat{\mathrm{u}}(\boldsymbol{w}, \mathrm{t})$.

iv. $F_{\alpha}\{\underbrace{T_{\alpha} \ldots T_{\alpha}(u(x, t))}_{n \text { times }}\}(w)=(i w)^{n} \widehat{u}(w, t), n=1,2,3, \ldots$

\section{Fourier sine and cosine transform}

In this subsection we shall discuss the Fourier sine and cosine transforms and some of their properties. These transforms are convenient for problems over semi-infinite and some of finite intervals in a spatial variable in which the function or its derivative is prescribed on the boundary.

\section{Infinite Fourier sine and cosine transform}

Definition 3 (Fourier cosine transform). The Fourier cosine Transform of a function $\mathrm{f}:[0, \infty] \rightarrow \mathrm{R}$ which is denoted by $\mathrm{F}_{\mathrm{c}}^{\alpha}(\mathrm{f}(\mathrm{t}))$ is defined as

$$
F_{c}^{\alpha}\{f(t)\}=\widehat{f}(w)=F_{c}^{\alpha}(w)=\sqrt{\frac{2}{\pi}} \int_{0}^{\infty} f(x) \cos \left(w \frac{x^{\alpha}}{\alpha}\right) x^{\alpha-1} d x .
$$

Definition 4 (Fourier sine transform). The Fourier sine Transform of a function $\mathrm{f}:[0, \infty] \rightarrow \mathrm{R}$ is defined as

$$
F_{s}^{\alpha}\{f(t)\}=\widehat{f}(w)=F_{s}^{\alpha}(w)=\sqrt{\frac{2}{\pi}} \int_{0}^{\infty} f(x) \sin \left(w \frac{x^{\alpha}}{\alpha}\right) x^{\alpha-1} d x .
$$

Lemma $4 \mathrm{~F}_{s}^{\alpha}$ and $\mathrm{F}_{\mathrm{c}}^{\alpha}$ are linear operators, i.e.,

$$
\begin{aligned}
& F_{c}^{\alpha}\{a f+b g\}=a F_{c}^{\alpha}\{f\}+b F_{c}^{\alpha}\{g\}, \\
& F_{s}^{\alpha}\{a f+b g\}=a F_{s}^{\alpha}\{f\}+b F_{s}^{\alpha}\{g\} .
\end{aligned}
$$

Theorem 4 Let $\mathrm{f}$ be a function defined for $\mathrm{t}>0$ and $\mathrm{f}(\mathrm{t}) \rightarrow 0$ as $\mathrm{x} \rightarrow \infty$. Then 
1. $F_{c}^{\alpha}\left(T_{\alpha}(f)(t)\right)=w F_{s}^{\alpha}(f(t))-\sqrt{\frac{2}{\pi}} f(0)$.

2. $F_{s}^{\alpha}\left(T_{\alpha}(f)(t)\right)=-w F_{c}^{\alpha}(f(t))$.

Proof. It can be easily proved by using Theorem 1 (f) and integration by parts.

\section{Finite Fourier sine and cosine transform}

When the physical problem is defined on a finite domain, it is generally not suitable to use transformation with an infinite range of integration. In such cases usage of finite Fourier transform is very advantageous.

Definition 5 The finite Fourier sine transform of $\mathrm{f}(\mathrm{t}), 0<\mathrm{t}<\mathrm{L}$ defined as

$$
F_{s}^{\alpha}\{f(t)\}=F_{s}^{\alpha}(n)=\int_{0}^{L} f(t) \sin \left(\frac{n \pi t^{\alpha}}{L^{\alpha}}\right) t^{\alpha-1} d t
$$

where $0<\alpha<1$.

The inverse Fourier sine transform is defined as follows,

$$
f(x)=\frac{2 \alpha}{L^{\alpha}} \sum_{n=1}^{\infty} F_{s}^{\alpha}(n) \sin \left(\frac{n \pi t^{\alpha}}{L^{\alpha}}\right) .
$$

Definition 6 The finite Fourier cosine transform of $\mathrm{f}(\mathrm{t}), 0<\mathrm{t}<\mathrm{L}$ defined as

$$
F_{c}^{\alpha}\{f(t)\}=F_{c}^{\alpha}(n)=\int_{0}^{L} f(t) \cos \left(\frac{n \pi t^{\alpha}}{L^{\alpha}}\right) t^{\alpha-1} d t
$$

where $0<\alpha<1$.

The inverse Fourier cosine transform is defined as follows,

$$
f(x)=\frac{\alpha}{L^{\alpha}} F_{c}^{\alpha}(0)+\frac{2 \alpha}{L^{\alpha}} \sum_{n=1}^{\infty} F_{c}^{\alpha}(n) \cos \left(\frac{n \pi t^{\alpha}}{L^{\alpha}}\right) .
$$

In bounded domain, the Fourier sine and cosine transforms are useful to solve PDE's. Therefore we can give following calculations.

$$
F_{s}^{\alpha}\left\{\frac{\delta^{\alpha} u}{\delta x^{\alpha}}\right\}=-\frac{n \pi \alpha}{L^{\alpha}} F_{c}^{\alpha}\{u(x, t)\},
$$




$$
\begin{aligned}
F_{s}^{\alpha}\left\{\frac{\delta^{\alpha}}{\delta x^{\alpha}} \frac{\delta^{\alpha} u}{\delta x^{\alpha}}\right\} & =-\frac{n \pi \alpha}{L^{\alpha}} F_{c}^{\alpha}\left\{\frac{\delta^{\alpha} u}{\delta x^{\alpha}}\right\} \\
& =-\frac{n^{2} \pi^{2} \alpha^{2}}{L^{2 \alpha}} F_{s}^{\alpha}\{u(x, t)\}-\frac{n \pi \alpha}{L^{\alpha}}[u(L, t) \cos n \pi-u(0, t)] .
\end{aligned}
$$

And then,

$$
\begin{aligned}
& F_{c}^{\alpha}\left\{\frac{\delta^{\alpha} u}{\delta x^{\alpha}}\right\}=\frac{n \pi \alpha}{L^{\alpha}} F_{s}^{\alpha}\{u(x, t)\}-[u(0, t)-u(L, t) \cos n \pi], \\
& F_{c}^{\alpha}\left\{\frac{\delta^{\alpha}}{\delta x^{\alpha}} \frac{\delta^{\alpha} u}{\delta x^{\alpha}}\right\}=-\frac{n^{2} \pi^{2} \alpha^{2}}{L^{2 \alpha}} F_{c}^{\alpha}\{u(x, t)\}-\frac{n \pi \alpha}{L^{\alpha}}\left[\frac{\delta^{\alpha} u(0, t)}{\delta x^{\alpha}}-\frac{\delta^{\alpha} u(L, t)}{\delta x^{\alpha}} \cos n \pi\right] .
\end{aligned}
$$

Now, let's apply this transform to solve space fractional heat equation,

$$
\frac{\delta u}{\delta t}=\frac{\delta^{\alpha}}{\delta x^{\alpha}} \frac{\delta^{\alpha} u}{\delta x^{\alpha}}, 0<x<L, t>0
$$

with the conditions,

$$
\begin{gathered}
u(L, t)=u(0, t)=0 \\
u(x, 0)=f(x)
\end{gathered}
$$

where $0<\alpha<1$.

When we apply the Fourier sine transform both sides of the equation, we have the following equality by using (14) and the conditions (16)

$$
\frac{d \widehat{u}(n, t)}{d t}=-\frac{n^{2} \pi^{2} \alpha^{2}}{L^{2 \alpha}} \widehat{u}(n, t) .
$$

Solving the above differential equation gives us,

$$
\widehat{u}(n, t)=C e^{-\frac{n^{2} \pi^{2} \alpha^{2}}{L^{2 \alpha}} t} .
$$

To evaluate $\mathbf{C}$, we apply Fourier sine transform to the condition (17). At the end we have $\mathrm{C}$ as,

$$
C=\widehat{u}(n, 0)=\int_{0}^{L} f(x) \sin \left(\frac{n \pi x^{\alpha}}{L^{\alpha}}\right) x^{\alpha-1} d x .
$$

Hence we get,

$$
\widehat{u}(n, t)=\left[\int_{0}^{L} f(x) \sin \left(\frac{n \pi x^{\alpha}}{L^{\alpha}}\right) x^{\alpha-1} d x\right] e^{-\frac{n^{2} \pi^{2} \alpha^{2}}{L^{2 \alpha}} t} .
$$


At the end applying inverse Fourier sine transform, the solution of Eq. (15) obtained as

$$
u(x, t)=\frac{2 \alpha}{L^{\alpha}} \sum_{n=1}^{\infty}\left[\int_{0}^{L} f(x) \sin \left(\frac{n \pi x^{\alpha}}{L^{\alpha}}\right) x^{\alpha-1} d x\right] e^{-\frac{n^{2} \pi^{2} \alpha^{2}}{L^{2 \alpha}} t} \sin \left(\frac{n \pi x^{\alpha}}{L^{\alpha}}\right) .
$$

\section{Conclusion}

In this paper we discuss about the solution of time and space fractional heat differential equations. Conformable fractional derivative definition is used for the solution time fractional heat equation. Conformable Fourier transform which will have very important role in fractional calculus like conformable Laplace transform is defined and given an application for space fractional heat equation. We can say that this definition has many advantages in the solution procedure of fractional differential equations. Some comparisons with classical fractional differential equations are given by Khalil and Abdeljawad before. This paper can help to see the researchers that given definitions are very helpful under the suitable conditions.

\section{References}

[1] K. Oldham, J. Spanier, The Fractional Calculus, Theory and Applications of Differentiation and Integration of Arbitrary Order, Academic Press, 1974.

[2] K. S. Miller, B. Ross, An Introduction to the Fractional Calculus and Fractional Differential Equations, A Wiley-Interscience Publication, 1993.

[3] I. Podlubny, Fractional Differential Equations, Academic Press, 1999.

[4] A. A. Kilbas, H. M. Srivastava, J. J. Trujillo, Theory and Applications of Fractional Differential Equations, Elsevier, 2006.

[5] N. Nyamoradi, Infinitely Many Solutions for a Class of Fractional Boundary Value Problems with Dirichlet Boundary Conditions, Mediterr. J. Math., 11 (2014), 75-87.

[6] R. Garrappa, M. Popolizio, Exponential Quadrature Rules for Linear Fractional Differential Equations, Mediterr. J. Math., 12 (2015), 219-244. 
[7] Y. Jalilian, R. Jalilian, Existence of Solution for Delay Fractional Differential Equations, Mediterr. J. Math., 10 (2013), 1731-1747.

[8] A. Esen, Y. Ucar, N. Yagmurlu, O. Tasbozan, A Galerkin finite element method to solve fractional diffusion and fractional diffusion-wave equations, Math. Model. Anal., 18 (2) (2013), 260-273.

[9] R. Khalil, M. Al Horani, A. Yousef, M. Sababheh, A new definition of fractional derivative, J. Comput. Appl. Math., 264 (2014), 65-70.

[10] M. Abu Hammad, R. Khalil, Conformable fractional heat differential equation, Int. J. Pure Appl. Math., 94 (2) (2014), 215-221.

[11] P. K. Kythe, P. Puri, M. R. Schäferkotter, Partial Differential Equations and Mathematica, CRC Press, 1997.

[12] T. Abdeljawad, On conformable fractional calculus, J. Comput. Appl. Math., 279 (2015) 57-66.

[13] N. T. Negero, Fourier transform methods for partial differential equations, Internat. J. Partial Differ. Equ. Appl., 2 (3) (2014), 44-57. 